\title{
DIAGNOSTIC MODALITIES FOR EARLY RECTAL CANCER - REVIEW OF THE LITERATURE
}

\author{
Valentin Ignatov, Nikola Kolev, Anton Tonev, Shteryo Shterev, Elitsa Encheva, \\ Aleksandar Zlatarov, Krasimir Ivanov
}

Department of Surgery, Medical university of Varna

\begin{abstract}
Rectal carcinoma represents a significant health problem in the modern world. Its high rate of occurrence and mortality in the late stages of the disease stresses the need for better diagnostic, prognostic and predictive methods. We present a review of the literature concerning the staging modalities, including the characteristics of early rectal carcinoma. Endoscopy, endoluminal ultrasound and magnetic resonance imaging play a major role in the preoperative staging and the restaging of rectal carcinoma. The new devices and technologies improve the diagnostic process, thus providing an option for new management strategies and better results.
\end{abstract}

Keywords: early rectal cancer, diagnostics, endoscopy, chromoendoscopy, endorectal ultrasonography, magnetic resonance

\section{INTRODUCTION}

Rectal carcinoma represents a significant health problem in the modern world. Its high rate of occurrence and mortality in the late stages of the disease stresses the need for better diagnostic, prognostic and predictive methods. The emergence of new data resulting from the researchers conducted on molecular level improved the understanding about the occurrence and progression of the tumours. The complexity and heterogeneity of the disease have been increased, which explains why so few biological markers find routine application in clinical practice. Sporadic cancer arises as a result of genetic and epigen-

\footnotetext{
Address for correspondence:

Anton Tonev

First Clinic of Surgery, University Hospital "St. Marina"

Medical University of Varna

$1 \mathrm{Hr}$. Smirnenski Str.

9000, Varna, Bulgaria

E-mail:teraton@abv.bg
}

Received: December 30, 2013

Accepted: February 11, 2014 etic changes, accumulated in the cell over the time of its life (1). These changes lead to a dysregulation of sensitive mechanisms, which in turn violates the normal cell proliferation and growth. Colorectal cancer develops randomly in most cases, although a small percentage of cases are a result of inherited mutations or inflammatory diseases of the colon (such as Crohn's disease and ulcerative colitis). According to modern oncological standards, the staging of rectal cancer depends on the time of diagnosis. The biological nature of the tumour is still not a subject of routine testing. According to previously introduced and used oncological standards, the most important factor, influencing the results of the operative treatment and survival rates, is the existence of metastasis $(2,3)$. No more than $6-8 \%$ of patients in the first stage of the disease present recurrence and progression with distant metastases in the lymph nodes and the liver. Widely used conventional methods and criteria for cancer staging estimate the external phenotypic expression, rate of progression of the neoplastic process- excessive growth, local invasion, metastasis, but do not give information about the minimal residual disease and its effects on survival rates (4). 
Valentin Ignatov, Nikola Kolev, Anton Tonev et al.

Rectal cancer starts its development from one cell or a group of cells that have lost their ability for controlled growth and integration with the functions of the macroorganism. Depending on the degree of development, it can be divided into the following subtypes: early- limited by the basal layer of the mucosa; invasive - passing through the basal layer of the mucosa and advanced- a cancer present with lymph node metastasis or distant metastasis.

According to the TNM classification, early cancer means carcinoma in situ, which is marked as Tis. Its frequency, according to different scientific studies varies from $0.2 \%$ to $11 \%$ (4) and increases courtesy of the screening programs. In an asymptomatic group of patients, older than 50 years, who have passed direct colonoscopy, the established frequency of adenocarcinoma is $1.8 \%$, of which $50 \%$ were carcinoma in situ. (5). Frequency between $3 \%$ and $4.6 \%$ has been reported from the conducted screening programmes after a positive immunologic fecal test for occult bleeding (1). In 2006, Rubio (18) reports 10 cases of patients with colon hyperplastic polyposis syndrome and presents a systematic review, demonstrating that $50 \%(70 / 147)$ of all patients present with this syndrome develop rectal cancer. Bearing in mind that cancerous cells most often develop from adenomatous cells, the main role for increasing the number of patients with early diagnosed cancer is the detection and removal of malignant polyps of the colon and rectum (6).

\section{Characteristics of Early Rectal Carcinoma}

Histology: Carcinoma in situ, intra-mucous carcinoma, high-grade dysplasia or intra-epithelial neoplasia is all stages of the disease, in which the muscularis propria layer is not involved. In general, cancer at this stage does not metastasise. It is classified as pTis or stage 0 according to the TNM classification. These terms are defined as non-invasive high-grade neoplasia according to the Vienna classification (6). Carcinoma in situ, high-grade dysplasia or intra-epithelial neoplasia corresponds to cancer, limited to the epithelial layer without invasion in to the lamina propria. Intra-mucous carcinoma invades the lamina propria layer. When the cancer extends to the sub-mucous layer, the polyp is considered malignant with a possibility of metastasising in lymph nodes or extra-regional localisation. Tumours, involving the sub-mucous layer are classified as T1 according to the TNM classification and correspond to the first clinical stage, provided that no regional or distant metastasis is present. (7) The term "pseudoinvasion" refers to cases in which glandular epithelium from the mucous layer is present under the muscularis propria layer of the colonic polyps. These lesions have no malignant potential and can be treated as adenomas (7). This phenomenon can be confused with invasive carcinoma by an inexperienced pathologist. Pseudo-invasion occurs in large polyps (over 1 $\mathrm{cm}$ ), especially in those with large peduncles and is most frequently seen in polyps localised in the sigmoid colon. Islands of adenomatous epithelium are localised in the muscularis mucosae layer and the submucous layer of the peduncle.

Morphology: The Paris classification (tab.1) divides the lesions based on morphological principles into polypoidal (pedunculated or sessile) and nonpolypoidal (flat or ulcerated) (fig.1) (8). The endoscopist should be familiar with the features of the lesion that reveal its malignant character. Such features are size, presence of ulcerative concaves, irregular contour, deformities, short and rigid peduncle, inability to lift a sessile polyp by infiltrating the submucous layer with serum. Non-polypoidal rectal neoplasms often develop into carcinoma (sub-mucous invasive carcinoma) compared to polypoidal neoplasms, regardless of size (4). Suspected lesions might be diagnosed using chromo-endoscopy and magnifying technique to highlight anomalies in the glandular structures and reveal the depth of the submucosal invasion (6). Kudo (8) classified colon polyps in six groups according to the findings of magnification endoscopy with indigo-carmine dye, complementing the diagnostic data before the endoscopic treatment. The endoscopic ultrasound, performed with endorectal ultrasound, endoscopic ultrasound or trans-endoscopic ultrasound miniprobes, is the most accurate method of assessing the submucous or deeper invasion in the colonic wall. This might help to send patients with deeper infiltration and higher risk of lymph node metastases directly to surgical treatment (12). 
Diagnostic modalities for early rectal cancer

Table 1. Paris classification of newly discovered neoplasms of the colon and rectum

\begin{tabular}{ll} 
Type of lesion & Characteristics \\
1. Type 0 -I & Polypoidal lesions \\
-(0-Ip) & Pedunculated lesion \\
-(0-Is) & Flat lesions \\
2. Type 0 -II & Non-raised and non-concave lesions \\
-(0-IIa) & Slightly raised \\
-(0-IIb) & Completely flat lesions \\
-(0-IIc) & Slightly concave lesions without ulcerations \\
3. Type 0 -III & Non-polypoidal lesions with ulcerations \\
\hline
\end{tabular}

The known prognostic factors are: invasion of the colonic wall, penetration to the mesorectal fascia (circumferential resection margin) and presence of extraregional metastases. Those factors are assessed by the methods of imaging diagnostics. The examinations, that give indications for local excision of early rectal carcinoma, are endorectal ultrasonography (ERUS) and magnetic resonance imaging (MRI). ERUS is the most accurate method for evaluation of tumour invasion to the rectal wall, especially for the early stages (T1-T2) (13).

ERUS can evaluate the penetration of the tumour in the mesorectal fatty tissue in T3 tumours, but cannot assess the circumferential resection margin. The rectal wall can be assessed also by MRI, as this method can establish penetration to the mesorectal fascia, although with lower accuracy for T1T2 tumours. Both methods have similar efficacy for evaluation of the regional lymph nodes. The ERUS can detect pathologically enlarged and hypoechoic lymph nodes and the MRI - lymph nodes over $1 \mathrm{~cm}$. The risk of lymph metastases is close to $0 \%$, although some new studies establish for tumours in Kakuchi stage sm 1 that the risk approximates $1 \%-3 \%$, for sm 2 it reaches $8 \%$ and in sm 3 - up to $23 \%$ (15). The selection of the patients for curative endoscopic resection MRI and ERUS must be performed before the procedure (16-18). The specificity of the endoscopic ultrasound devices allows for endoluminal localisation of the colonic tumours. Some authors report results of ultrasound examination of neoplasms of the colon with significant specificity and sensitivity. The early rectal carcinoma can be treated with the methods of endoscopic surgery (14). The treatment of colorectal cancer has developed significantly in the recent years and the exact preoperative staging plays an important role in the choice of the optimal multimodal strategy. Prognostic factors such as the depth of tumour invasion (T-stage) and the number of engaged lymph nodes (N-stage) is easily evaluated by ERUS. Correct staging is crucial to properly select the patients, the needed preoperative therapy and to choose the optimal surgical method (5). The contemporary methods for preoperative staging of colorectal cancer include computer tomography (CT), endorectal ultrasonography (ERUS), magnetic-resonance imaging (MRI), positrone-emission tomography (PET). Choosing the imaging modality is defined by its availability in the medical centre and the experience with its use (6). Since the introduction of endorectal ultrasound (ERUS) in the early 80 's $(6,7)$ it has had a wide use in the everyday clinical practice as it plays important role in the diagnostic and staging of the malignant neoplasms of the gastro-intestinal tract and the adjacent structures. ERUS was applied in rectal cancer staging in 1985 with promising results (9) and now it is accepted as a method for diagnostics of rectal tumours, being considered a quick, safe method with high staging accuracy (10). The depth of invasion in the rectal wall and the adjacent structures is a powerful predictive factor for survival and plays an important role in the choice of the treatment strategy. The accuracy of the T-staging varies according to different literature data. A retrospective study over a 10 -year period reports of $69 \%$ overall accuracy for Tstaging (11). The validation of ERUS staging was done 
Valentin Ignatov, Nikola Kolev, Anton Tonev et al.

by comparing the pathological stage of the resected tumours. T3 tumors were staged with higher accuracy (86\%) and the differentiation between $\mathrm{T} 1$ and $\mathrm{T} 2$ stage was considered especially difficult. Overstaging was common (19\%) and in only $12 \%$ understaging was observed. The authors notice high variability between the different physicians and correlation of higher accuracy with better experience. When comparing different frequencies, $10 \mathrm{MHz}$ gives better results than $7.5 \mathrm{MHz}$ but only in T1 tumours. Other authors report of $81 \%$ accuracy in preoperative T-staging in a series of 424 patients, where the examination was performed by experienced surgeons (12). In a prospective multicentre study of application of ERUS in clinical practice the overall accuracy was $68.5 \%$, less than the previously reported data, probably due to the lack of experience (16). The highest sensitivity was achieved for T3 tumours (74.9\%). Meta-analysis for the period 1980-2008 demonstrated high sensitivity of ERUS in the evaluation of the tumour invasion (88\%-95\%) and better results were achieved in the more advanced stages of the disease (13).

In rectal cancer it is very important to differentiate between the early and the advanced stage. In a prospective study the selection of patients was performed according to the ERUS-stage and the achieved accuracy was 95\% (18). Another group of authors reports of $96 \%$ sensitivity, $85 \%$ specificity and $94 \%$ accuracy in the differentiation of early and advanced rectal cancer. A meta-analysis strongly recommends ERUS for the staging of early tumours, because of its excellent sensitivity and specificity in the diagnostics of T0 stage $(97.3 \%$ and $96.3 \%$, respectively) (20). Although the results vary, a common tendency is established in the abovementioned studies. The accuracy of endorectal ultrasonographic staging varies for the T-stage and the uT2 tumours are diagnosed most often (13). This is a result of overstaging. The better results depend on the physician's experience $(11,21)$. For patients with stenosing tumours the staging is suboptimal, because the ERUS probe cannot pass through. In these cases despite the advanced stage of the tumour a catheter ultrasound miniprobe is recommended (22), or dilatation of the tumour, followed by ERUS.

\section{N-stage}

A significant disadvantage of ERUS for routine staging of rectal carcinoma is its low accuracy in the evaluation of regional lymph nodes, which varies between $70 \%$ and $75 \%$ (22.23). Retrospective study presented unsatisfactory results in $68 \%$ overall accuracy in $\mathrm{N}$-staging, $52 \%$ sensitivity and $82 \%$ specificity (14). This confirms previous results from other groups who reported 10 years with $64 \%$ accuracy in the diagnosis of lymphatic metastases (15). A review of 35 studies over two decades, gave the following results: $73.2 \%$ pooled sensitivity, specificity $75.8 \%$ (16). Another conclusion of this meta-analysis is that ERUS is doing better in the exclusion of lymphatic metastases than in their establishment. One reason for the faulty interpretation of the image, leading to poor diagnostic results is the presence of altered reactive inflammatory lymph nodes. It is difficult to distinguish from malignant lymph nodes based only on the ultrasound characteristics, leading to false-positive results, the problem being the size of the nodes. While positive lymph nodes are generally round, hypoechogenic and over $5 \mathrm{~mm}$ in diameter (16-20) as metastatic foci were reported in approximately $18 \%$ of the nodes below 5 millimeters. One study that deserves attention found that the frequency of metastatic lymph nodes was $9.5 \%$ for $\leq 2 \mathrm{~mm}$ nodes in the shortest diameter, $47 \%$ for nodes between 3 and $5 \mathrm{~mm}$ and $87 \%$ in nodules $\geq 6 \mathrm{~mm}$ (14). A new study examines the accuracy of the determination of ERUS lymph stage correlated with depth of invasion of primary tumour.

In less invasive tumours the size of lymph nodes and metastatic sites, the accuracy of the method decreases (17). The ERUS ability to correctly identify lymph status decreased significantly from $84 \%$ for pT3 tumours to $48 \%$ in pT1 tumours, in which case the average size of the nodes as metastatic sites was respectively $3.3 \mathrm{~mm}$ and $0.3 \mathrm{~mm}$. In conclusion, early tumours have small metastatic foci that are released more frequently in ERUS. This may be the cause of pelvic recurrence after local excision. The logical question is whether patients with early cancer should be indicated for local excision only with ERUS staging. One solution to this problem is to reduce the size as a criterion. This can increase the responsiveness of 
the method for the establishing of the lymphatic metastases, but will lead to a lower overall accuracy and specificity. When the limit is $5 \mathrm{~mm}$, responsiveness, specificity and accuracy in diagnosing ERUS T1 lesions is about $38 \%, 94 \%$ and $89 \%$ while with the lowering of the limit to $3 \mathrm{~mm}$, the values were $75 \%, 49 \%$ and $53 \%$ (13).

The development and improvement of new technologies make endoscopic ultrasonography method of great clinical significance in diseases of the digestive system in terms of diagnosis, staging and prognosis (16). These new techniques are applicable to rectal tumors. The three-dimensional ultrasound (3D-ERUS) improves the study of spatial relations of rectal cancer and better staging and assessment of the respective. You can use linear or radial transducer. Three-dimensional reconstruction is achieved more easily with radial than with linear transducer while manual control and transducer angulation can lead to artifacts (17). Therefore, 3D-ERUS is a more accurate method than the two-dimensional CT and 2D-ERUS in the staging of rectal cancer. The accuracy of 3D-ERUS, 2D-ERUS and CT in assessing the depth of tumour infiltration was $78 \%, 69 \%$ and $57 \%$, while the evaluation of lymph status was $65 \%, 56 \%$ and $53 \%$. The most common cause of misinterpretation of the image is human error. 3D-ERUS provides more information about the depth of invasion and shows conical growths along the deep border of the tumour, which correlates well with the histological evidence of the degree of infiltration, more advanced T stage and lymph metastases (20).

Special software facilitates the manipulation with the rectal probe and makes $3 \mathrm{D}$ reconstruction easier. These 3D images provide better visualisation of mesorectal borders, thereby overcoming one of the 2D ultrasonography (17). 3D-ERUS contributes to the endoscopic mucosal resection of early tumours being a safer and more effective procedure (15). Elastrography is an innovation in the endoscopic ultrasound and explores the elastic properties of tissues, and this way it may contribute to differences between the malignant lesions of fibrous tissue and the benign ones. Preliminary data suggest that the combination of elastography with ERUS improves T-staging in rectal cancer. This method simulates a virtual palpation, and can thus identify lymph nodes that are more likely to be malignant and to increase the diagnostic accuracy of the ERUS fine needle biopsy. Contrast-enhanced endoscopic ultrasonography is a modern method, which uses contrast in the bloodstream, increasing the Doppler signal, which facilitates the evaluation of tumour perfusion. Although this method has not yet been fully explored, it is expected to become the method of functional diagnostics for the evaluation of response to neoadjuvant therapy. Apart from high resolution, this method provides information on changes in tumour vascularisation. Transfer of recognised principles for ultrasound staging of rectal tumours with topical study of lesions of the colon can help to improve results of radical surgical treatment of rectal cancer.

\section{Future direction}

Findings from the recent analyses did not lead to a revolution in rectal cancer. Constructed hypotheses must be validated by different methods of analysis before recommendations are made for the clinical use of biomarkers. The latest technological advance is the introduction of the method of sequential analysis. The identification of variants and mutations in individual tumours revealed certain tumorspecific abnormalities. They may find clinical use as prognostic and predictive factors, and also help the development of individualised therapeutic strategy. The increasing number of therapies targeting specific genes and signalling pathways emphasises the role of the normal functioning of genes to achieve the desired effect. With the better identification and validation of biomarkers, they will lead to a greater range of choice of therapy. Optimisation of the predictive methods for the development of cancer diagnosis at an early stage, giving the most correct prediction, prediction of therapeutic response - these are of great importance to patients, medical staff, but also to socio-economics to run.

\section{CONCLUSION}

Endoscopic ultrasonography has been demonstrated as an accurate method for local staging of rectal cancer. Despite its limitations, a wider application in clinical practice would improve treatment outcomes. New methods like 3D-ERUS, elastography and contrast enhancement would provide additional data to improve the diagnostic accuracy of endoscop- 
ic ultrasound to expand its role, to make its use routine in the complex treatment of colorectal cancer. While performing colonoscopy there may also occur complications. Since endoscopy has been playing an increasing therapeutic role, there will probably be an increase in the number of complications. Knowing the potential endoscopic complications, the expected frequency and risk factors help to reduce the number of complications. Endoscopy must conduct a careful selection of patients in the planned procedure to be familiar with the plan of procedure and equipment, and be prepared to deal with possible accidents. In the event of a complication there should be done an early diagnosis and intervention to reduce morbidity and mortality rate associated with this complication.

\section{REFFERENCES}

1. Rembacken BJ, Fujii T, Cairns A et al (2000) Flat and depressed colonic neoplasms: a prospective study of 1000 colonoscopies in the UK. Lancet 355:1211-1214

2. Hurlstone DP, Cross SS, Adam I et al (2003) A prospective clinicopathological and endoscopic evaluation of flat and depressed colorectal lesions in the UK. Am J Gastroenterol 98:2543-2549

3. Suzuki N, Talbot IC, Saunders BP. (2004) The prevalence of small, flat colorectal cancers in a western population. Colorectal Dis 6:15-20

4. Tsuda S, Veress B, Toth E et al (2002) Flat and depressed colorectal tumours in a southern Swedish population: a prospective chromoendoscopic and histopathological study. Gut 51:550-555

5. Saito Y, Waxman I, West AB et al (2001) Prevalence and distinctive biological features of flat colorectal adenomas in a North American population. Gastroenterology 120:1657-1665

6. Deyhle P, Largader F, Jenny S et al (1973) Amethod for endoscopic electroresection of sessile colonic polyps. Endoscopy 5:38-40

7. Rembacken BJ, Gotoda T, Fujii T et al (2001) Endoscopic mucosal resection. Endoscopy 33:709-718

8. Kudo S, Tamegai Y, Yamano $\mathrm{H}$ et al (2001) Endoscopic mucosal resection of the colon: the Japanese technique. Gastrointest Endosc Clin N Am 11:519-535

9. Kudo S, Tamura S, Kashida $\mathrm{H}$ et al (1996) Endoscopic treatment in colorectal lesionsespecially on endoscopic mucosal resection. Nippon Rinsho 54:1298-1306

10. Hurlstone DP, Cross SS, Brown S et al (2004) A prospective evaluation of high-magnification chromoscopic colonoscopy in predicting completeness of EMR. Gastrointest Endosc 59:642-650

11. Brooker JC, Saunders BP, Shah SG et al (2002) Treatment with argon plasma coagulation reduces recurrence after piecemeal resection of large sessile colonic polyps: a randomized trial and recommendations. Gastrointest Endosc 55:371-375

12. Lambert R, Provenzale D, Ectors $\mathrm{N}$ et al (2001) Early diagnosis and prevention of sporadic colorectal cancer. Endoscopy 33:1042-1064

13. Inoue $\mathrm{H}$, Takeshita $\mathrm{K}$, Hori $\mathrm{H}$ et al (1993) Endoscopic mucosal resection with a cap-fitted 
panendoscope for esophagus, stomach, and colon mucosal lesions. Gastrointest Endosc 39:58-62

14. Rembacken BJ, Fujii T, Kondo H. (2001) The recognition and endoscopic treatment of early gastric and colonic cancer. Best Practice Res Clin Gastroenterol 15:317-336

15. Fujishiro M, Yahagi N, Nakamura M et al (2006) Endoscopic submucosal dissection for rectal epithelial neoplasia. Endoscopy 38:493-497

16. Machida H, Sano Y, Hamamoto Y et al (2004) Narrow-band imaging in the diagnosis of colorectal mucosal lesions: a pilot study. Endoscopy 36:1094-1098

17. Chiu HM, Chang CY, Chen CC et al (2006) A prospective comparative study of narrow-band imaging, chromoendoscopy and conventional colonoscopy in the diagnosis of colorectal neoplasia. Gut Sep 27

18. Kudo S, Rubio CA, Teixeira CR et al (2001) Pit pattern in colorectal neoplasia: endoscopic magnifying view. Endoscopy 33:367-373

19. Saitoh Y, Obara T, Einami K et al (1996) Efficacy of high-frequency ultrasound probes for the preoperative staging of invasion depth in flat and depressed colorectal tumors. Gastrointest Endosc 44:34-39

20. Palazzo MGL, Canard PJM (2001) Guidelines of the French Society of Digestive Endoscopy: endoscopic mucosectomy. Endoscopy 33:187-190 